\title{
Chronic necrotising pneumonia caused by Aspergillus niger
}

\author{
J WIGGINS, T J H CLARK, B CORRIN \\ From the Brompton Hospital, London
}

\begin{abstract}
A woman with asthma developed chronic necrotising semi-invasive pneumonia due to mixed Aspergillus niger and Candida albicans infection; though not severely immunosuppressed, she may have been predisposed by long term oral corticosteroid and recurrent oral antibiotic treatment. The diagnosis should be considered in patients with chronic airflow limitation who develop cavitating pneumonia.
\end{abstract}

Rapidly disseminating invasive aspergillosis occurs in severely debilitated or immunosuppressed individuals. Less well recognised is the more indolent, semi-invasive chronic necrotising form of pulmonary aspergillosis. ${ }^{1-3}$ It is characterised by cavitation and is liable to be confused with the better known aspergilloma, particularly as the newly formed cavity may contain a ball like sequestrum of heavily infected necrotic lung tissue.

The species found most commonly in all forms of pulmonary aspergillosis is Aspergillus fumigatus. A flavus is less commonly responsible, and human disease resulting from infection by $A$ niger is rare.

\section{Case report}

A 57 year old woman, a smoker, developed asthma at the age of 33 years and required long term oral corticosteroid treatment to control symptoms; the lowest dose of prednisolone she took was $6 \mathrm{mg}$ daily. Skinprick test responses to A fumigatus were negative in 1986. In September 1987 the asthma was reasonably well controlled and she had no other complaints. The FEV, was 0.6 litres, and the forced vital capacity 1.451 , and full blood count and results of serum biochemical investigations were normal.

Two weeks later she developed anorexia and weight loss with purulent sputum production. A chest radiograph was normal. Amoxycillin treatment was ineffective and she was admitted four weeks later with persistent purulent sputum, high fever, and further weight loss. Chest radiography showed a cavitating lesion in the right upper lobe with surrounding consolidation (fig 1). The white blood cell count was $20.3 \times 10^{9} / 1(95 \%$ neutrophils) but no organisms were identified in numerous sputum samples. The results of the following investigations were normal or negative: sputum cytology for malignant cells, viral antibodies, cold aggluti-

Address for reprint requests: Professor B Corrin, Brompton Hospital, London SW3 6HP.

Accepted 9 February 1989

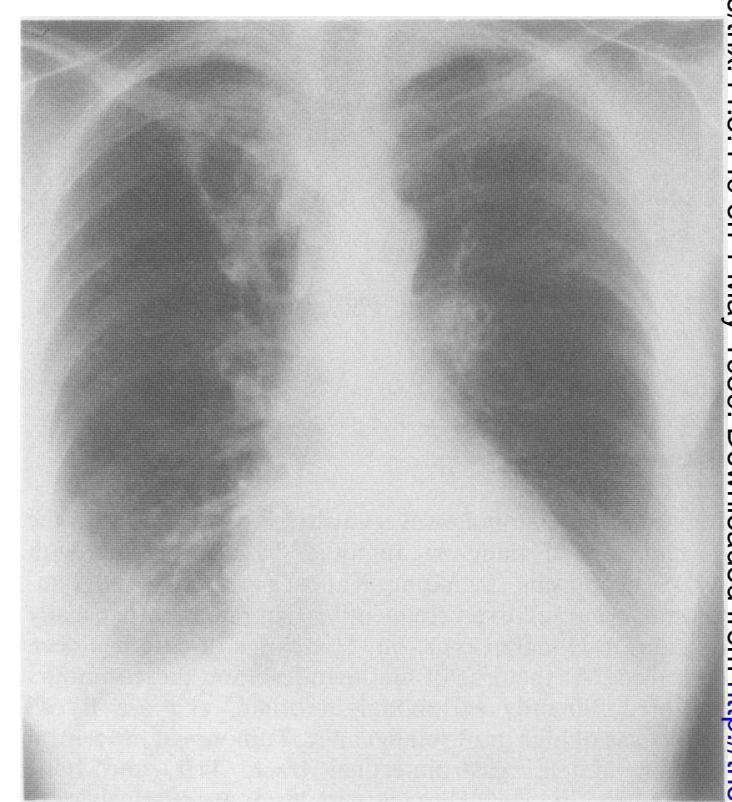

Fig 1 Chest radiograph showing a cavitating lesion in the right upper lobe.

nins, antibodies to Legionella pneumophila and Mycoplasma pneumoniae, and skinprick test responses and precipitins to $A 3$ fumigatus. Treatment was changed to penicillin, metronidazole, and flucloxacillin. The prednisolone was increased to $40 \mathrm{mg}$ daily.

Fibreoptic bronchoscopy showed no endobronchial lesion and washings and brushings yielded no abnormalities. $N$ Biopsy showed severely inflamed and partly necrotic bron- or chus, neutrophil infiltration of alveolar walls, and a separate $N$ fragment composed of fungal hyphae, tentatively identified N as Aspergillus. Itraconazole was added to her treatment. The $\sigma$ patient was unfit for surgery and despite intensive physiotherapy deteriorated and died eight days after admission. The $\frac{C}{\square}$ total duration of her terminal illness was $\mathbf{3 5}$ days.

At postmortem examination a $3 \mathrm{~cm}$ diameter cavity in the $\stackrel{\mathscr{\infty}}{+}$ right upper lobe was seen to be surrounded by extensive $\square$ consolidation. The cavity was partly lined by plaques of a $O$ white mould (fig 2), culture of which yielded a moderate growth of $A$ niger and a heavy growth of Candida albicans. Microscopy of the plaques showed a dense tangle of aspergillus hyphae and candida spores. The cavity lacked an epithelial lining and fibrous capsule. Its wall was formed of a $?$ 


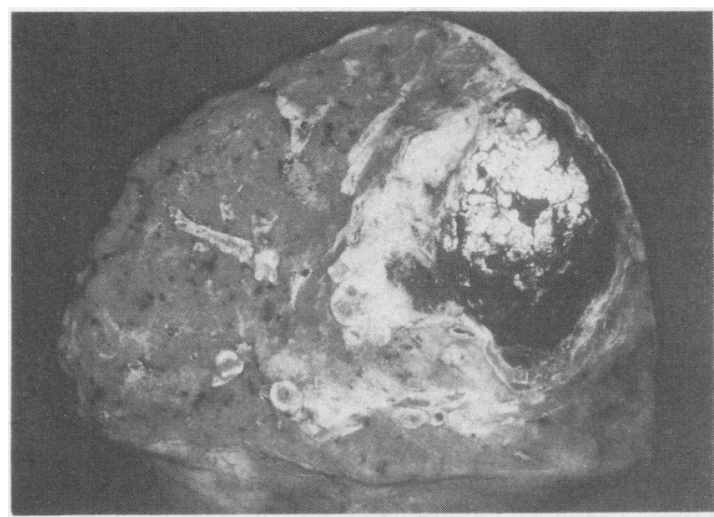

Fig 2 Right upper lobe with a cavity partly lined by a chalky white plaque of fungus and surrounded by a zone of consolidation. Though white in this non-ventilated cavity, the fungus was black when cultured in air and proved to be Aspergillus niger.

$1 \mathrm{~cm}$ band of necrotic lung tissue infiltrated by aspergillus hyphae and candida spores. Outside this, consolidated viable lung tissue contained many candida spores. The consolidation was largely fibrinous and neutrophils were sparse. There was no evidence of thrombosis or vascular invasion by either fungus. There was heavy calcium oxalate crystal deposition, particularly in artery walls and on the side of the vessel nearest the cavity. There was no tissue eosinophilia, either in relation to the infection or in bronchi distant from the cavity, but the bronchi showed other changes consistent with asthma-hypertrophy of bronchial muscle and glands and thickening of the basement membrane of the surface epithelium. There was no evidence of disseminated aspergillosis or oxalosis.

\section{Discussion}

This patient illustrates the poorly recognised condition of semi-invasive pulmonary aspergillosis' or chronic necrotising aspergillus pneumonia. ${ }^{2}$ Most cases of such pneumonia are caused by $A$ fumigatus; in only two of 25 previously reported patients was $A$ niger responsible, as in our case, and in one of these $A$ fumigatus was also present. ${ }^{12}$ Calcium oxalate crystal deposition was prominent in our case and is a recognised feature of infection by all species of Aspergillus, but in particular $\boldsymbol{A}$ niger. ${ }^{4}$ The fungus produces oxalic acid, which precipitates as insoluble calcium oxalate crystals.

The cavities that develop in chronic necrotising aspergillus pneumonia frequently contain ball like collections resembling aspergillomas. ${ }^{2}$ These collections often comprise heavily infected necrotic lung tissue and the term sequestrum is preferable to aspergilloma. ${ }^{5}$ In this and other cases, a pure colony of fungus is found in the cavity with invasion of the adjacent lung. This primary local invasion distinguishes chronic necrotising aspergillus pneumonia from aspergilloma. The latter is a saprophytic colonisation of a preformed cavity, whereas in the former the cavity is newly formed and a consequence of the infection.

The role of the associated candida infection in our patient is uncertain. Virus infections, such as influenza, may predispose to invasive aspergillosis, ${ }^{6}$ but in our patient there was no evidence of prior virus infection.

Our case emphasises that chronic necrotising aspergillus pneumonia may occur in patients who do not have severe immunosuppression, though like many others-a quarter of one series ${ }^{2}$ - she was taking long term oral corticosteroids. This treatment in conjunction with recurrent courses of systemic antibiotics may predispose patients with chronic airflow limitation to this indolent form of invasive aspergillosis. The diagnosis therefore needs to be considered in patients with chronic airflow limitation who develop cavitating pneumonia. Unfortunately, the diagnosis may be difficult to establish as tests for aspergillus precipitins may give negative results, ${ }^{2}$ particularly in patients taking oral corticosteroids, and the relevance of fungi in sputum is difficult to assess in patients having long term antibiotics.

Chronic necrotising aspergillus pneumonia carries a high mortality rate ${ }^{3}$ and our therapeutic measures were unsuccessful. Early treatment with systemic amphotericin with or without flucytosine may be successful, ' but greater experience with these agents is needed in this disorder.

We are indebted to Dr R Hay for his invaluable advice.

\section{References}

1 Gefter WB, Weingrad TR, Epstein DM, Ochs RH, Miller WT. "Semi-invasive" pulmonary aspergillosis. A new look at the spectrum of Aspergillus infections of the lung. Radiology 1981;140:313-21.

2 Binder RE, Faling LJ, Pugatch RD, Mahasaen C, Snider GL. Chronic necrotizing pulmonary aspergillosis: a discrete clinical entity. Medicine (Baltimore) 1982;61:109-24.

3 George PJM, Boffa PBJ, Naylor CPE, Higenbottam TW. Necrotising pulmonary aspergillosis complicating the management of patients with obstructive airways disease. Thorax 1983;38: 478-80.

4 Nime FA, Hutchins GM. Oxalosis caused by Aspergillus infection. Johns Hopkins Med J 1973;133:183-94.

5 Kibbler CL, Milkins SR, Bhamru A, Spiteri M, Noone P, Prentice HG. Apparent pulmonary mycetoma following invasive aspergillosis in neutropenic patients. Thorax 1988;43:108-12.

6 Horn CR, Wood NC, Hughes JA. Invasive aspergillosis following post-influenza pneumonia. Br J Dis Chest 1983;77:407-10.

7 Rodenhuis S, Beaumont F, Kauffman HF, Sluiter HJ. Invasive pulmonary aspergillosis in a non-immunocompromised patient: successful management with systemic amphotericin and flucytosine and inhaled amphotericin. Thorax 1984;39:78-9. 